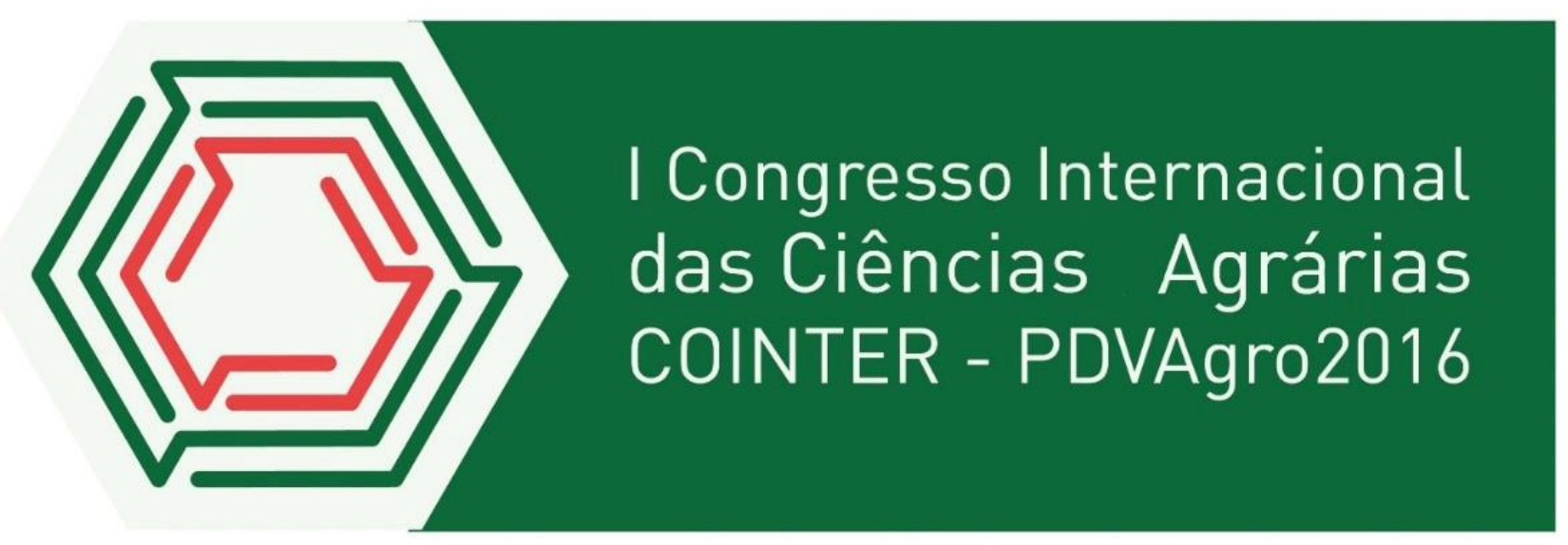

\title{
DETERMINAÇÃO DE MO NO SOLO PELA OTIMIZAÇÃO DA REAÇÃO CATALÍTICA DE KI + $\mathrm{H}_{2} \mathrm{O}_{2}$ EM ESPECTROFOTOMETRIA
}

\author{
Apresentação: Comunicação Oral
}

Larissa Grasiela de Arruda Ferreira Costa ${ }^{1}$; Lyllian Brunelly do Santos ${ }^{2}$; Nayara Rose da Conceição Lopes $^{3}$; Renato Lemos dos Santos ${ }^{4}$

\section{Resumo}

O Mo, um elemento traço do solo é um micronutriente requerido para as plantas, sendo absorvido em sua forma iônica, molibdato. Apesar de sua importância, os seus efeitos na produtividade são pouco estudados. Isso, também, pela dificuldade na sua determinação. A determinação de Mo no solo pode ser feita pelo método catalítico da reação do $\mathrm{KI}+\mathrm{H}_{2} \mathrm{O}_{2}$, associada a extratores ácidos, potencializada pelo Brij, para leitura em espectrofotômetro. Assim, o objetivo foi avaliar diferentes tempos de reação e influência de distintas concentrações de cada reagente no processo catalítico do $\mathrm{KI}+\mathrm{H}_{2} \mathrm{O}_{2}$, bem como as soluções extratoras, na reação da determinação em Mo disponível no solo. Para isso, avaliou-se o efeito das soluções extratoras de cloreto de potássio $(\mathrm{KCl})$, sulfato de sódio (Na2SO4) ambos a $1 \mathrm{~mol} / \mathrm{L}-1$, e uma solução duplo ácida, o Mehlich-1, avaliou-se o tempo de reação $(2,4,8,16$ e $32 \mathrm{~min})$, assim como foi analisada a reação de oxidação com diferentes concentrações para os reagentes de trabalho, o NH4F e KI, respectivamente $(0,00 ; 0,10 ; 0,20 ; 0,40$ e $0,80 \%)$ e $\mathrm{H}_{2} \mathrm{O}_{2}(0,00 ; 0,010 ; 0,020 ; 0,040$ e $0,080 \%)$. A solução extratora de

\footnotetext{
'Bacharelado em Agronomia, Instituto Federal de Educação, Ciência e Tecnologia de Pernambuco - Campus Vitoria de Santo Antão, grasielalala@gmail.com

Técnica em Química/ Licenciada em Física, Instituto Federal de Educação, Ciência e Tecnologia de Pernambuco Campus Vitoria de Santo Antão, Iyllian.santos@vitoria.ifpe.edu.br

${ }^{3}$ Bacharelado em Agronomia, Instituto Federal de Educação, Ciência e Tecnologia de Pernambuco - Campus Vitoria de Santo Antão, nayara_rose1@hotmail.com

${ }^{4} \mathrm{Dr}$. em Ciências do Solo/ docente/ coordenador do Departamento de pesquisa e extensão, IFPE - Campus Vitória de Santo Antão, renato.santos@vitoria.ifpe.edu.br
} 
Mehlich-1, por apresentar meio ácido, foi mais constante nos valores de absorbância. O tempo de reação mais adequado se assemelha ao padrão da metodologia analisada. As concentrações mais adequadas para os reagentes $\mathrm{NH}_{4} \mathrm{~F}, \mathrm{KI}$ e $\mathrm{H}_{2} \mathrm{O}_{2}$ estão no intervalo entre 0,1 e $0,2 \%$; entre 0,1 e $0,2 \%$ e entre 0,01 e $0,02 \%$ respectivamente.

Palavras-Chave: Molibdênio, reações químicas, espectrofotometria.

\section{Introdução}

O Mo, micronutriente encontrado naturalmente em pequenas concentrações no solo, de até $100 \mathrm{mg} \mathrm{kg}^{-1}$ sem prejudicar o crescimento vegetal (YUSRAN, 1993). Nas plantas, desempenha principalmente função de ativador enzimático. Na maioria dos solos o Mo disponível está na forma de $\mathrm{MoO}_{4}{ }^{2-}$ e é conhecido por participar de reações redox que se encontra no solo. Geralmente, a baixa disponibilidade de Mo, que é observada em solos ácidos $(\mathrm{pH}<5,0)$ com a elevada presença de óxidos

de Fe e Al, levam as culturas exploradas sobre eles a se apresentarem deficientes em Mo (BRENNAN \& BOLLAND, 2007; FITRI et al., 2008).

Mesmo com conhecimento da importância do Mo para as plantas, a utilização deste micronutriente em adubações para fins de nutrição vegetal ainda é escassa. Assim, também se encontram dificuldades quanto ao uso de uma solução extratora mais adequada na determinação de seus níveis disponíveis no solo.

Segundo Bezerra e Ferreira (2006) diferentes métodos analíticos que podem ser empregados para a determinação de Mo, são comumente sujeitos a interferências químicas. Um método eficiente e pouco oneroso é a determinação do Mo pelo método catalítico da reação do $\mathrm{KI}+\mathrm{H}_{2} \mathrm{O}_{2}$ e leitura em espectrofotômetro, foi um procedimento basicamente desenvolvido e automatizado para a determinação de Mo em amostras geológicas e biológicas por Fuge (1970). Este método tem sido utilizado com sucesso para a determinação do Mo em materiais vegetais (PLANK, 1992). Fontes et. al. (2000) ao afirmar que o ânion catalisa reação de iodeto de potássio (KI) e peróxido de hidrogênio $\left(\mathrm{H}_{2} \mathrm{O}_{2}\right)$ e formas de iodo molecular.

Neste contexto, o objetivo deste trabalho é avaliar a interferência da solução extratora e do tempo de reação e ainda a influência de distintas concentrações de cada reagente no processo catalítico do $\mathrm{KI}+\mathrm{H}_{2} \mathrm{O}_{2}$ na reação da determinação em Mo disponível no solo.

\section{Fundamentação Teórica}

O molibdênio nos vegetais tem função primordial de ativador enzimático, como de regular da atividade da redutaze do nitrato ( $\mathrm{ARN}$ ), enzima responsável pela redução do $\mathrm{NO}_{3}{ }^{-}$a $\mathrm{NO}_{2}{ }^{-}$na rota de 
assimilação do $\mathrm{N}^{-\mathrm{NO}_{3}}{ }^{-}$. Além disso, o Mo atua como regulador a atividade da nitrogenase, enzima responsável pela redução do $\mathrm{N}_{2}$ a $\mathrm{NH}_{3}$ em organismos que realizam a fixação biológica de $\mathrm{N}$ (FBN). Desta maneira pode-se associar a disponibilidade deste micronutriente no solo ao aumento da absorção de $\mathrm{N}$ e de produtividade, pela correlação positiva entre a redutase do nitrato ou a nitrogenase (Santos, 2014).

A utilização deste micronutriente em adubações para fins de nutrição vegetal ainda é escassa, principalmente no cultivo da cana-de-açúcar. Consequentemente métodos de determinação ainda apresentam dificuldades, tanto para o método e aparelho utilizado quanto à solução extratora adequada na determinação de seus níveis disponíveis no solo. As concentrações do Mo no solo podem ser determinadas em espectrômetro de absorção atômica. Entretanto, os baixos níveis disponíveis não são detectados ao menos que o aparelho esteja acoplado em forno de grafite. Apesar de eficiente, essa determinação é pouco acessível devido ao elevado custo. A determinação de Mo em solução também pode ser realizado por espectrometria de emissão atômica por plasma acoplado, contudo, não para as concentrações de Mo disponíveis no solo, que se encontram abaixo do nível de detecção do método (Santos, 2012).

Quando se trata do uso de extratores para a determinação dos teores de elementos disponíveis no solo, a planta é a melhor maneira para representar a extração. Entretanto, os extratores químicos não conseguem extrair do solo as mesmas quantidades que as plantas, mas os valores obtidos por esses extratores podem se correlacionar com o extraído pela planta (Santos, 2012).

Os extratores que são utilizados para a determinação do Mo no solo são comumente extratores ácidos, como o oxalato ácido de amônio com pH 3,3 e o Mehlich-1 com pH entre 1,0 e 0,5, que conseguem retirar Mo em formas disponíveis do solo (Santos, 2012; Cantini et. al., 1970).

Fontes et al. (2000) ao comparar os extratores Mehlich-1 e Mehlich-3 para a determinação de Mo em solos brasileiros, observaram boa correlação entre cada método de extração. Segundo os autores há uma relação entre as características físicas, químicas e mineralógicas com a adsorção da partícula de Mo no solo.

\section{Metodologia}

Desenvolvida no Instituto Federal de Educação, Ciência e Tecnologia de Pernambuco (IFPE) - Campus Vitória de Santo Antão, a pesquisa é caracterizada pelas avaliações separadas de cada passo do processo reativo, o tempo de reação e a influência de cada um dos reagentes e ainda foram avaliados a influência dos extratores na solução

A metodologia padrão para determinação de Mo consiste, inicialmente, o preparo de soluções com água deionizada, com concentrações $0 ; 0,05 ; 0,08 ; 0,10 ; 0,15 ; 0,20 \mathrm{mg} \mathrm{L}^{-1}$ de Mo, preparados de 
acordo com metodologia descrita por Santos (2012). Posteriormente foram preparados os três reagentes utilizados no processo catalítico de determinação, o NH4F 0,25\%, pesando-se 0,25 g para $100 \mathrm{~mL}$ de solução; o $\mathrm{H}_{2} \mathrm{O} 2$ 0,02\%, onde pipetou-se 0,600 mL, de $\mathrm{H}_{2} \mathrm{O} 230 \%$, para $1.000 \mathrm{~mL}$ de solução, preparados com Brij, segundo Fontes et al. (2000) deve-se acrescentar 12 gotas de Brij por litro dos reagentes; e ainda o KI a 0,25\%, pesando-se 0,25 g para $100 \mathrm{~mL}$ de solução. Para a leitura das soluções padrões em espectrofotômetro, se utilizam $3 \mathrm{~mL}$ de cada solução, sendo adicionado 1 $\mathrm{mL}$ de cada reagente na ordem de preparo descrita acima, com um intervalo de um minuto para cada amostra, esperando-se 10 min de reação após a adição do último reagente. Para a leitura da absorbância foi utilizado espectrofotômetro com comprimento de onda de $420 \mathrm{~nm}$.

Considerando a metodologia padrão foi avaliado o efeito do tempo de reação e a utilização de cada reagente no processo reativo, em cinco ensaios analíticos separados. O primeiro ensaio, realizado no teste de reação de extratores foi realizado com três tipos de solução extratora diferentes, dois sais, o cloreto de potássio $(\mathrm{KCl})$, sulfato de sódio $\left(\mathrm{Na}_{2} \mathrm{SO}_{4}\right)$ ambos a $1 \mathrm{~mol} / \mathrm{L}-1$, e uma solução duplo ácida, o Mehlich-1, a padrão. Nestas soluções foi realizada a determinação de $\mathrm{pH}$, em três repetições, para fortalecimento da discussão do processo reativo. Para extração de Mo disponível foram colocados $10 \mathrm{~cm}^{-3}$ de solo em erlenmeyers e $100 \mathrm{ml}$ de cada extrator mais $1 \mathrm{~g}$ de carvão ativado, sendo agitados em mesa orbital por 15 min e posto para decantar por 16 horas. Posteriormente, o sobrenadante foi filtrado e armazenado para a determinação.

Para o segundo ensaio, utilizou-se cinto tempos de reação, $(2,4,8,16$ e $32 \mathrm{~min})$ preparados com Mehlich-1, utilizando-se de quatro repetições. O processo de extração foi realizado com solos coletados da Estação Experimental de Cana-de-açúcar de Carpina. As amostras coletadas foram levadas para o laboratório de Química e Fertilidade do Solo do IFPE - Campus Vitoria de Santo Antão, colocados para secar ao ar durante aproximadamente sete dias, sendo depois destorroados e tamisadas em peneira de malha $2 \mathrm{~mm}$, obtendo-se a terra fina seca ao ar (TFSA). Após obtenção da TFSA, procedeu-se a extração do Mo disponível, com a agitação de 100 mL de Mehlich-1 com 10 $\mathrm{cm}^{-3}$ de solo e $1 \mathrm{~g}$ de carvão ativado, por $15 \mathrm{~min}$. Dezesseis horas depois, o sobrenadante foi filtrado e coletado para determinação do teor de Mo. Para os três demais ensaios foi realizado um novo processo de extração do Mo disponível seguindo a mesma metodologia já descrita. No terceiro e quarto ensaio foram analisadas cinco concentrações do $\mathrm{NH} 4 \mathrm{~F}$ e $\mathrm{KI}$, respectivamente $(0,00 ; 0,10 ; 0,20 ; 0,40$ e $0,80 \%)$. No quinto ensaio foram testadas concentrações de $\mathrm{H} 2 \mathrm{O} 2$ $(0,00 ; 0,010 ; 0,020 ; 0,040$ e $0,080 \%)$. Em todos os ensaios foram utilizadas quatro repetições por análise.

Após as análises, os dados foram submetidos à análise de variância. Quando se observou efeito significativo (Teste $\mathrm{f}, \mathrm{p}<0,05$ ) foi realizada análise de regressão, sendo selecionado o modelo que 
melhor representa o fenômeno, aquele com maior valor de coeficiente de determinação $\left(\mathrm{R}^{2}\right)$. Quando se observaram efeitos significativos, nos dados de solução extratora, as suas médias foram comparadas pelo teste de Tukey ao nível de $5 \%$ de probabilidade

\section{Resultados e Discussões}

No ensaio da reação com diferentes extratores de solo, o tempo de reação interferiu significativamente sob a ação dos extratores (Tabelas 1).

Tabela 1: Valores de absorbância em espectrofotômetro a $420 \mathrm{~nm}$ em diferentes tempos de reação e tipos de extratores

\begin{tabular}{cccc}
\hline Tempo & \multicolumn{3}{c}{ Extrator } \\
\cline { 2 - 4 } & Mehlich-1 & KCL & $\mathrm{NA}_{2} \mathrm{SO}_{4}$ \\
\hline----- min---- & Absorbância \\
2 & $0,026 \mathrm{dA}$ & $0,012 \mathrm{bB}$ & $0,013 \mathrm{aB}$ \\
5 & $0,038 \mathrm{cA}$ & $0,015 \mathrm{abB}$ & $0,006 \mathrm{bC}$ \\
10 & $0,035 \mathrm{cA}$ & $0,012 \mathrm{bB}$ & $0,005 \mathrm{bC}$ \\
15 & $0,043 \mathrm{bA}$ & $0,001 \mathrm{cC}$ & $0,013 \mathrm{aB}$ \\
20 & $0,058 \mathrm{aA}$ & $0,019 \mathrm{aB}$ & $0,006 \mathrm{bC}$ \\
\hline
\end{tabular}

Fator

Tempo

Extrator

Tempo*Extrator

$\mathrm{CV}(\%)$
$38,3573 * *$

$990,4106 * *$

$49,3140 * *$

12,10

de Mo disponível do solo

Letras minúsculas iguais na linha e maiúsculas na coluna não diferem pelo teste de Tukey; ${ }^{\text {ns }}$ não significativo; $*$, **, *** significativos, aos níveis de $5 \%, 1 \%$ e $0,1 \%$ de probabilidade, respectivamente.

Independente do tempo de reação, os valores de absorbância foram maiores com a solução de

\begin{tabular}{ccc}
\hline Mehlich-1 & $\mathrm{KCL}$ & $\mathrm{Na}_{2} \mathrm{SO}_{4}$ \\
\hline \multirow{2}{*}{0,23} & 4,72 & 3,08 \\
\hline & Desvio Padrão & \\
\hline 0,03 & 0,01 & 0,06
\end{tabular}

Mehlich-1 (Tabela 2). Isso provavelmente ocorreu devido aos extratores KCL e $\mathrm{Na}_{2} \mathrm{SO}_{4}$ não 
possuírem pH tão ácido quanto a solução de Mehlich-1 (Tabela 2), não desencadeando a reação com os reagentes e desenvolvimento da coloração do comprimento de onda de $420 \mathrm{~nm}$.

Tabela 2. Valores de pH para os três extratores na determinação de Mo disponível do solo.

Nos valores de absorbância com o extrator Mehlich-1 houve crescimento com os tempos de reação (Tabela 3). Fontes et. al. (2000) indicaram que o Mehlich-1 é o extrator mais adequado para extração dos teores de Mo disponível no solo. Entretanto, quando se utilizou os extratores KCL e $\mathrm{Na}_{2} \mathrm{SO}_{4}$, os valores de absorbância não apresentaram um comportamento padrão. Com a solução de KCL, o valor da absorbância aos 5 min se assemelhou ao demais, exceto aos 15 min. Com o $\mathrm{Na}_{2} \mathrm{SO}_{4}$, os valores de absorbância aos 2 min de reação assemelharam-se aos $15 \mathrm{~min}$, e as leituras aos 5 min foi semelhante aos 10 e 20 minutos. Esse comportamento, provavelmente, foi devido as diferenças de $\mathrm{pH}$ das soluções (Tabela 2).

De acordo com Fontes et. al. (2000) e Santos (2012), a reação de determinação de Mo em solo por espectrofotometria ocorre em meio ácido. Os valores de absorbância em função do tempo de reação se ajustaram ao modelo linear, provavelmente devido ao crescente e proporcional efeito do tempo de reação no processo catalítico.

No teste do tempo de reação foi observado que entre os tempos de 2 a 8 min houve pequena variação de leituras. Esse comportamento possivelmente aconteceu devido a rapidez da leitura e tendo o processo reativo se completasse (Figura 1). Entretanto, aos 16 e 32 min de reação, os valores foram maiores, presumindo-se assim que o processo catalítico da reação após os 10 min, valor padrão, já passe para sucessivos processos degradativos.

Nas avaliações com os reagentes de trabalho, os valores de absorbância do $\mathrm{NH}_{4} \mathrm{~F}$ e $\mathrm{H}_{2} \mathrm{O} 2$ se adaptaram ao modelo de regressão exponencial decrescente (Figuras 2 e 3). Os valores absorbância com $0 \%$ de $\mathrm{NH}_{4} \mathrm{~F}$ apresentaram-se os mais altos. Da concentração de 0 a $0,1 \%$, os valores de absorbância decairam, chegando a quase zero com $0,2 \%$. Nas concentrações maiores, 0,4 e $0,8 \%$ os valores de absorbância igualaram-se a zero.

Um comportamento semelhante ao do $\mathrm{NH}_{4} \mathrm{~F}$ foi observado no ensaio do $\mathrm{H}_{2} \mathrm{O}_{2}$, onde os valores de absorbância decresceram com o aumento das concentrações de $\mathrm{H}_{2} \mathrm{O}_{2}$ (Figura 3). Este decaimento de valores de absorbância com o aumento das concentrações demonstra, possivelmente, que o KI não consegue realizar a catálise em maiores porcentagens de $\mathrm{NH}_{4} \mathrm{~F}$ e $\mathrm{H}_{2} \mathrm{O}_{2}$.

Apenas com o aumento da concentração de KI se observaram valores crescentes de absorbância no processo reativo, por isso adequou-se melhor o modelo linear crescente (Figura 4). As concentrações avaliadas mostraram que no processo reativo o KI apresenta função de catalizador, como demonstra Fontes et al. (2000) em estudos realizados na extração de Mo do solo. Quando a 
concentração foi de $0 \%$ de KI, não há valores de significativos de absorbância. Nos valores de 0,1 e $0,2 \%$ de KI, os valores de absorbância não foram muito distantes, o que possivelmente demonstra que em 10 minutos o processo catalítico ocorre de forma constante.

Quando as concentrações foram mais altas, de 0,4 e 0,8\%, no mesmo intervalo de tempo, o processo reativo passa por uma catalise maior e apresenta menor leitura devido discrepância de valores obtidos na absorbância. Assim, é possível afirmar que só ocorre catálise na reação na presença do KI.

Figura 1: Valores de absorbância em espectrofotômetro a 420 nm em diferentes tempos de reação em Mehlich-1.

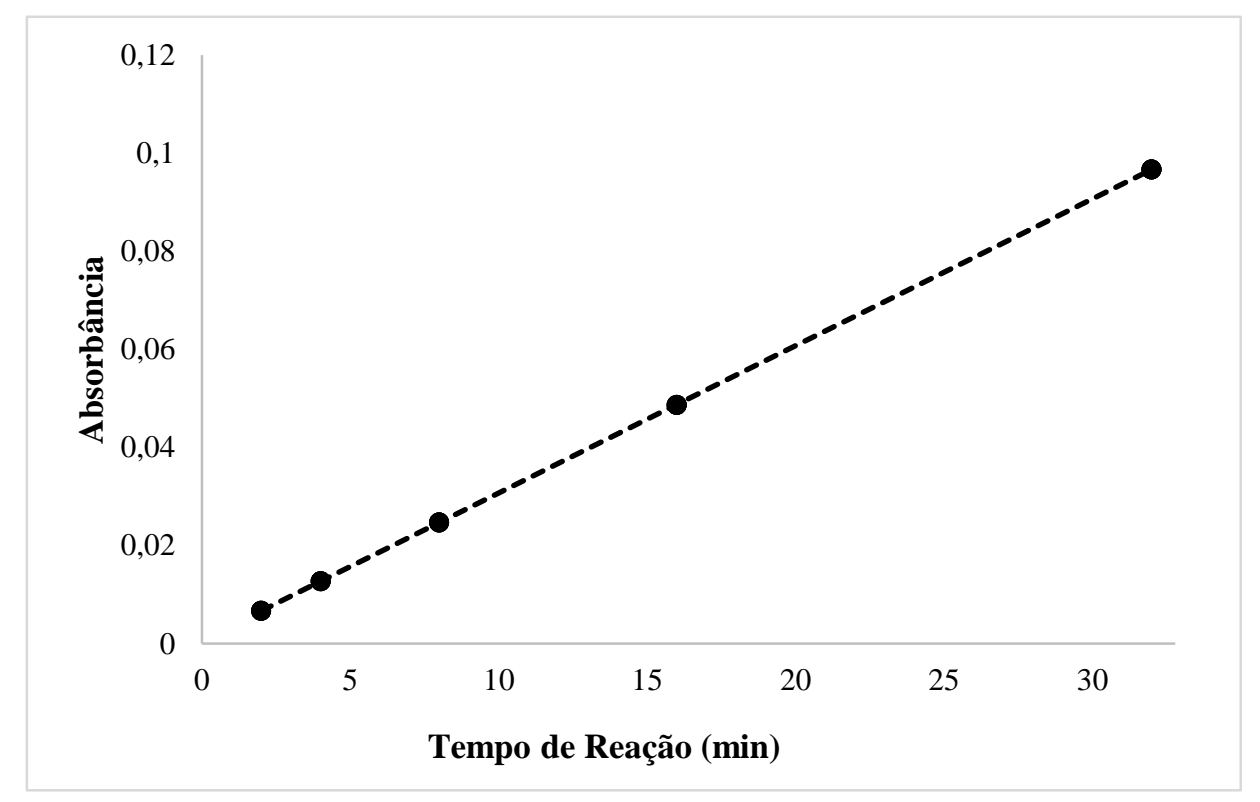

Figura 2: Valores de absorbância em espectrofotômetro a $420 \mathrm{~nm}$ em diferentes concentrações de $\mathrm{NH}_{4} \mathrm{~F}$, no processo reativo do $\mathrm{KI}+\mathrm{H}_{2} \mathrm{O}_{2}$ em extrato de Mehlich-1.

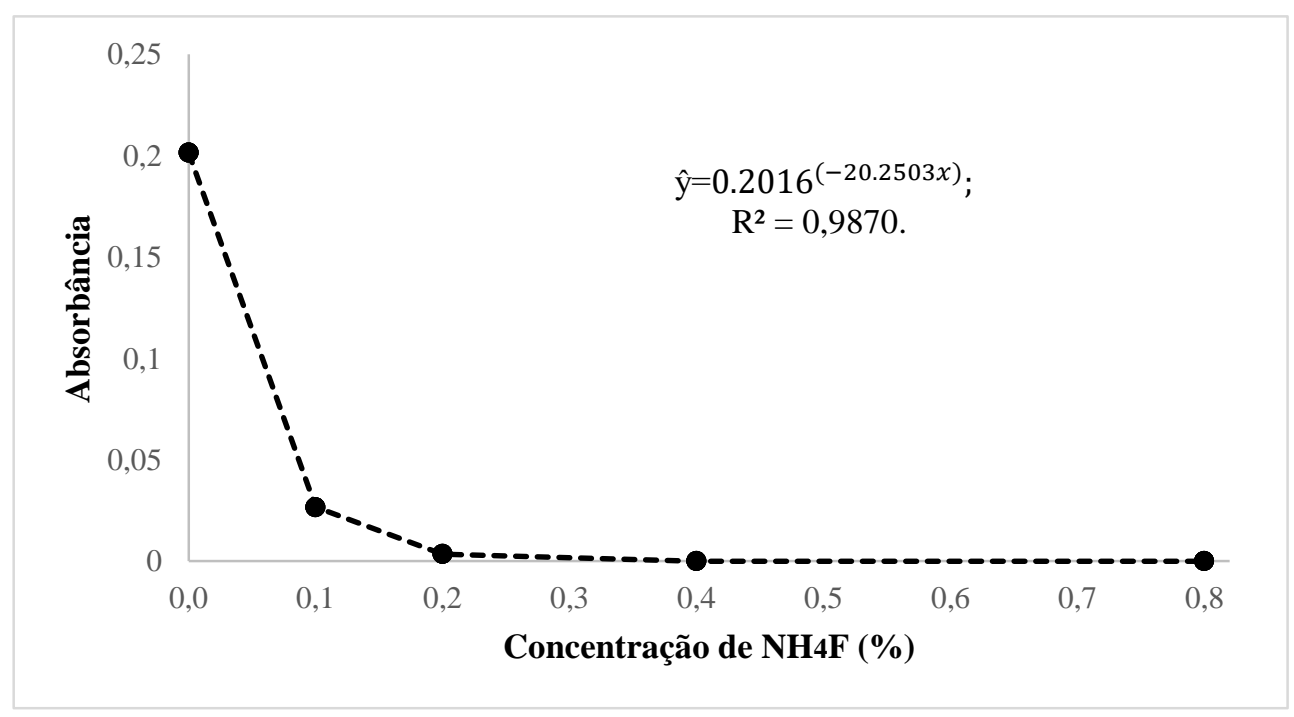


Figura 3: Valores de absorbância em espectrofotômetro a $420 \mathrm{~nm}$ em diferentes concentrações de $\mathrm{H}_{2} \mathrm{O}_{2}$, no processo reativo do $\mathrm{KI}+\mathrm{H}_{2} \mathrm{O}_{2}$ em extrato de Mehlich-1

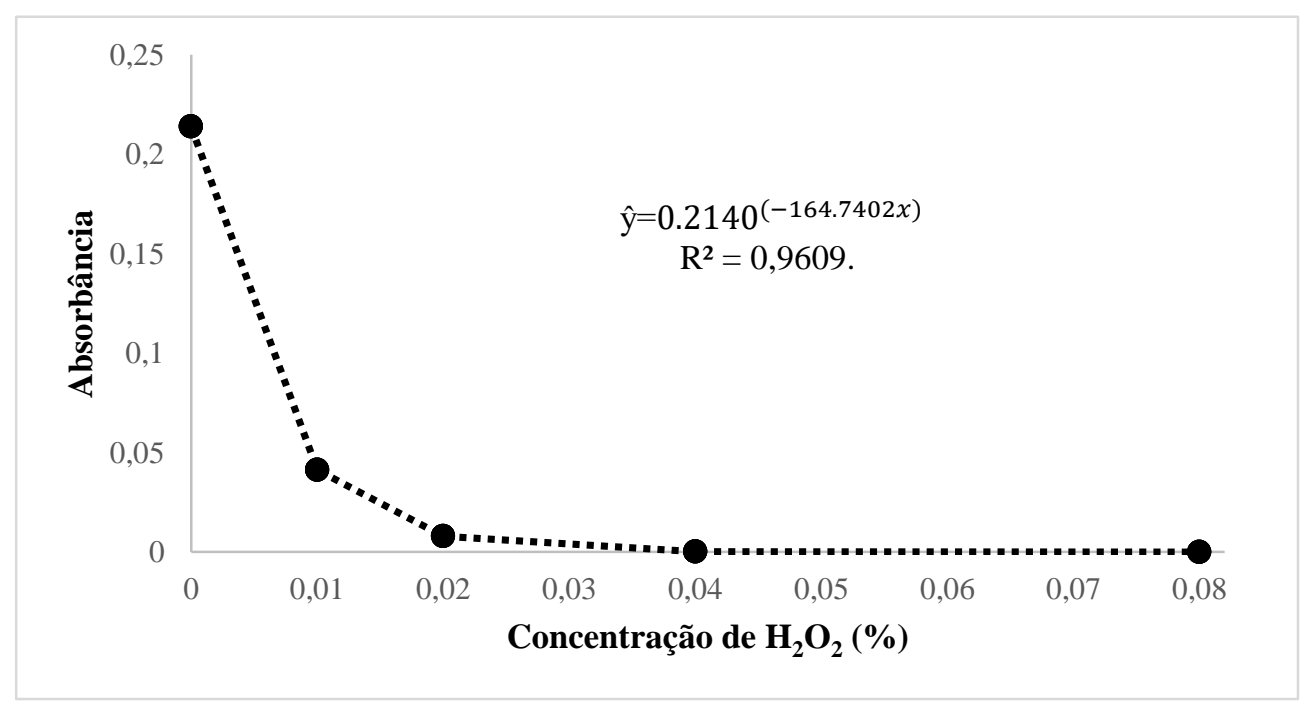

Figura 4: Valores de absorbância em espectrofotômetro a $420 \mathrm{~nm}$ em diferentes concentrações de KI, no processo reativo do $\mathrm{KI}+\mathrm{H}_{2} \mathrm{O}_{2}$ em extrato de Mehlich-1

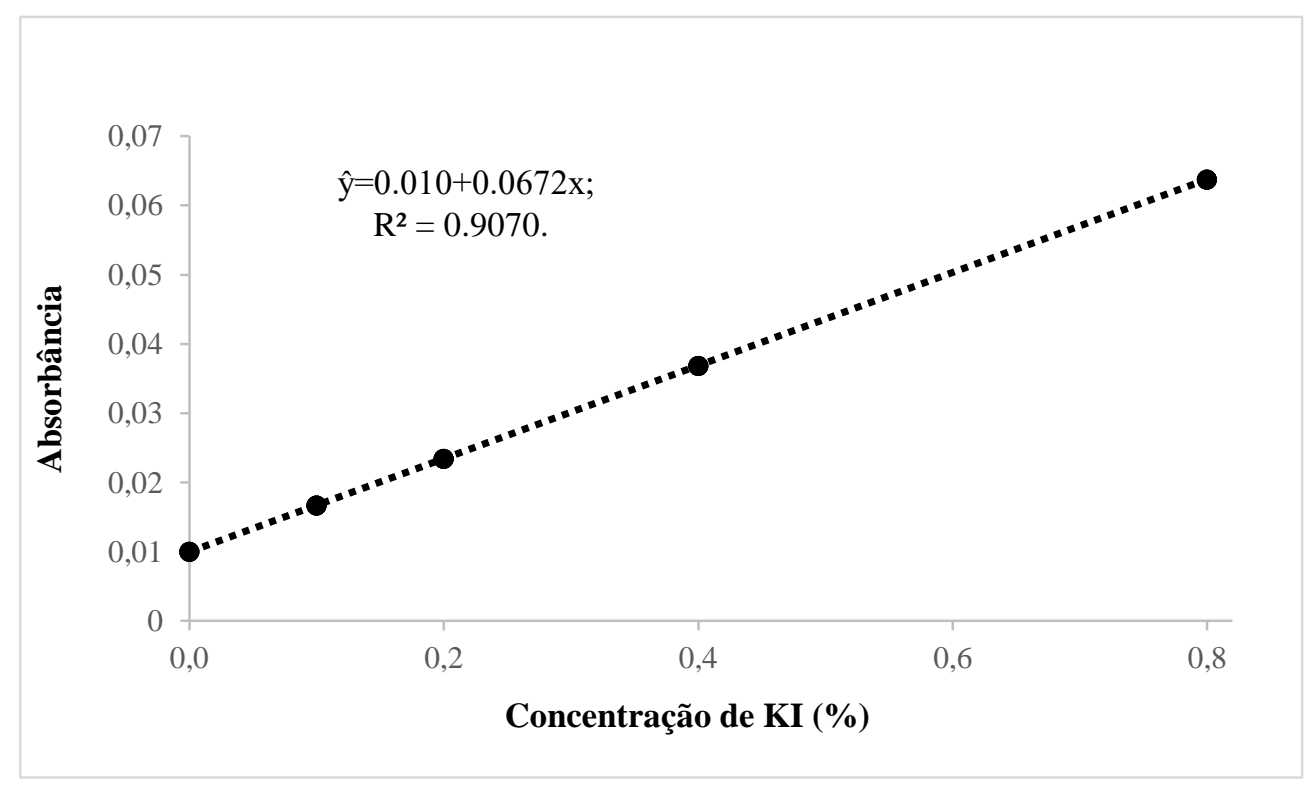

\section{Conclusões}

Apenas o extrator Mehlich-1, por apresentar meio ácido, apresentou um padrão de reação.

O tempo de reação mais adequado se assemelha ao padrão da metodologia analisada.

As concentrações mais adequadas para os reagentes $\mathrm{NH}_{4} \mathrm{~F}, \mathrm{KI}$ e $\mathrm{H}_{2} \mathrm{O}_{2}$ estão no intervalo entre 0,1 e $0,2 \%$; entre 0,1 e $0,2 \%$ e entre 0,01 e $0,02 \%$ respectivamente. 


\section{Referências}

BEZERRA M. A., FERREIRA, S.L.C., Extração em ponto nuvem: Princípios e aplicações em química analítica. Edições UESB, Vitória da Conquista, 2006.

BRENNAN, R. F.; BOLLAND, M. D. A. Increased Concentration of Molybdenum in Sown Wheat Seed Decreases Grain Yield Responses to Applied Molybdenum Fertilizer in Naturally Acidic Sandplain Soils. Journal of Plant Nutrition, v. 30, n. 12, p. 2005-2019, 3 dez. 2007.

CATANI, R. A.; ALCARDE, J. C.; FURLANI, P. R. A determinação do teor total e do teor solúvel, em diversas soluções, de molibdênio do solo. Anais da Escola Superior de Agricultura Luiz de Queiroz, v. 27, p. 171-180, 1970.

FITRI, N. et al. Molybdenum Speciation in Raw Phloem Sap of Castor Bean. Analytical Letters, v. 41, n. 10, p. 1773-1784, 16 jul. 2008.

FONTES, R. L. F. ;DALLPAI, D.L. ; BRAGA, J. M. E ALVAREZ, V., V. H. Determination of molybdenum in soil test extracts with potassium iodide plus hydrogen peroxide reaction. Communications in Soil Science and Plant Analysis, V. 31: 15, 2671 - 2683, 2000.

FUGE, R. An automated method for the determination of molybdenum in geological and biological samples. Analyst, v. 95, n. 1127, p. 171-176, 1970.

PLANK, O.C. Plant Analysis reference procedures for the southern region of the united states southern cooperative series bulletin 368,Crop \& Soil Science.

SANTOS, Marilúcia de Jesus. Extração e determinação de Mo em três argissolos do nordeste cultivados com cana-de-açúcar. Recife: UFRPE, 2012. 80 p. Dissertação (Mestrado) - Programa de Pós- Graduação em Ciência, Universidade Federal Rural de Pernambuco, Recife, 2012.

SANTOS, R.L. Molibdênio no metabolismo e na fixação biológica de nitrogênio em cana-de-açúcar. Universidade Federal Rural de Pernambuco - Recife. 135p. Tese de Doutorado - Programa de PósGraduação em Ciência do Solo, 2014.

YUSRAN,F.H.Triple superphosphate and urea effects on availability of nutrients in the fertilizer band for soybean (glycine max 1.) Tese de Doutorado.. Growth witr emphasis on molybdenum,1993. 